\title{
The old nova DQ Her (1934)
}

\section{The eclipse of the triple-peaked $\mathrm{H}_{\alpha}$ profile}

\author{
A. Bianchini1 ${ }^{1,2}$, E. Mastrantonio ${ }^{2,3}$, R. Canterna ${ }^{2}$, J. Stute $^{2}$, and K. Cantrell ${ }^{2}$ \\ 1 University of Padova, Department of Astronomy, vicolo dell'Osservatorio 2, 35122 Padova, Italy \\ e-mail: bianchini@pd.astro.it \\ 2 Department of Physics and Astronomy, University of Wyoming, Laramie, WY 82071, USA \\ 3 On leave from Lycoming College, Williamsport, PA, USA
}

Received 1 June 2004 / Accepted 7 July 2004

\begin{abstract}
We performed time resolved spectroscopy of the old-nova intermediate-polar DQ Her during an eclipse of its WD primary. The eclipse of the $\mathrm{H}_{\alpha}$ and $\mathrm{HeI} \lambda 6678$ emission lines is reminiscent of that of an accretion disk. However, $\mathrm{H}_{\alpha}$ shows a triplepeaked profile and is only partially eclipsed. The three stationary peaks shown by $\mathrm{H}_{\alpha}$ could be produced by the accretion stream overflowing the disk, some material near the barycenter of the binary system, the surface of the secondary facing the WD, a wind and/or a jet from the disk. Some contamination by the nova ejecta is also possible. The presence of stationary components in the emission line profiles is reminiscent of other CVs like the dwarf nova IP Peg. The broad component of the $\mathrm{H}_{\alpha}$ emission seems to be produced by the accretion disk as this component is the only one to show radial velocity variations modulated by the orbital motion of the WD. The $\mathrm{O}-\mathrm{C}$ value derived from the eclipse of the continuum confirms the cyclical behavior of the $\mathrm{O}-\mathrm{C}$ vs. JD diagram and suggests a main secular periodicity slightly longer than $\sim 22 \mathrm{yr}$, possibly due to magnetic activity cycles of the secondary.
\end{abstract}

Key words. stars: novae - cataclysmic variables - accretion, accretion disks - stars: white dwarfs - stars: magnetic fields

\section{Introduction}

Classical novae $(\mathrm{CNe})$ belong to the class of Cataclysmic Variables (CVs) and are formed by a white dwarf (WD) primary accreting matter from a Roche lobe filling companion, usually a low mass main sequence star. The WD is surrounded by an accretion disk, unless its magnetic field is strong enough to partially or totally control the accretion geometry, these two cases corresponding to the Intermediate Polar systems (hereafter IPs) and the Polar systems, respectively. At any given orbital period, the luminosities of old novae are systematically brighter than those of dwarf novae (DN), suggesting that the former are powered by larger mass transfer rates from the secondary $\left(\dot{M}_{2}\right)$ and/or have hotter WDs preventing the onset of the disk instability phenomena observed in DN. Another difference between novae and dwarf novae is represented by the requirement that the primaries of nova systems must have masses larger than $\sim 0.6 M_{\odot}$ so the accreted material is cyclically ejected through nova outbursts (Livio 1992).

DQ Her is one of the most studied classical novae. It brightened in 1934 reaching $m_{V}=1.3$ while its post outburst magnitude is $m_{V} \sim 14.3$ (Duerbeck 1987, 1999); the distance is 400 pc (Duerbeck 1999). DQ Her is the prototype of those slow novae which display a dip in their decline light curve due to dust formation. It is also the prototype of intermediate polar binary systems (IP) since the discovery of the $71 \mathrm{~s}$ light modulation (Walker 1954, 1956). The main characteristics of all DQ Her systems have been reviewed by Patterson (1994). According to the model discussed by Chanan et al. (1978), a beam of X-ray and UV photons originating at the polar caps of the rotating WD moves around illuminating the surface of the concave disk where the $71 \mathrm{~s}$ optical pulses originate. The $71 \mathrm{~s}$ stable pulsations are also observed in the HeII $\lambda 4686$ line (Chanan et al. 1978; Martell et al. 1995), in the UV continuum and several UV emission lines (Silber et al. 1996b), but not in the X-ray band (Silber et al. 1996a; Mukai et al. 2003). In particular, DQ Her is a surprisingly faint X-ray source (Orio et al. 2001). The eclipse light curve of the old nova has been systematically studied by Walker (1956), Nather \& Warner (1969), Nelson \& Olson (1976) and Schneider \& Greenstein (1979). The most recent ephemeris of the eclipsing binary (Zhang et al. 1995; Ogloza et al. 2000) suggest a period of $4.6469 \mathrm{~h}$.

Although DQ Her is an eclipsing binary system, several parameters of this widely studied old nova remain uncertain. The first radial velocity measurements of DQ Her suggested both high-mass solutions with $M_{1} \sim 1.1 M_{\odot}$ and $M_{2} \sim 0.55 M_{\odot}$ (Robinson 1976; Hutchings et al. 1979), and low-mass 
Table 1. The journal of the observations.

\begin{tabular}{lll}
\hline \hline Spectrum & $\begin{array}{l}\text { HJD(mid - exposure) } \\
245]\end{array}$ \\
& $2452413+$ & \\
\hline s1 & 0.5099 & 0.79 \\
s2 & 0.5240 & 0.87 \\
s3 & 0.5285 & 0.89 \\
s4 & 0.5331 & 0.91 \\
s5 & 0.5376 & 0.94 \\
s6 & 0.5421 & 0.96 \\
s7 & 0.5467 & 0.98 \\
s8 & 0.5512 & 1.01 \\
s9 & 0.5558 & 1.03 \\
s10 & 0.5603 & 1.06 \\
s11 & 0.5650 & 1.08 \\
s12 & 0.5696 & 1.10 \\
s13 & 0.5741 & 1.13 \\
s14 & 0.5787 & 1.15 \\
s15 & 0.5832 & 1.17 \\
16 & 0.5878 & 1.20 \\
s17 & 0.5923 & 1.22 \\
s18 & 0.5969 & 1.24 \\
\hline
\end{tabular}

solutions with $M_{1} \sim 0.47 M_{\odot}$ and $M_{2} \sim 0.34 M_{\odot}$ (Smak 1980; Young \& Schneider 1980). Finally, direct spectroscopic measurements of the cool secondary star allowed Horne et al. (1993) to the more reliable estimates $M_{1} \sim 0.60 M_{\odot}, M_{2} \sim$ $0.40 M_{\odot}$ and $i \sim 86.5^{\circ}$; such a mass of the WD is required for a system exhibiting slow nova outbursts (Starrfield 1989). Horne et al. (1993) also give $K 1=140 \mathrm{~km} \mathrm{~s}^{-1}, K 2=227 \mathrm{~km} \mathrm{~s}^{-1}$, $i=86.5^{\circ} V_{D} \sim 800 \mathrm{~km} \mathrm{~s}^{-1}, R_{D} / R_{L 1} \sim 1$, and a total eclipse width $\Delta \Phi=0.22 P$.

In this paper we present the results of time-resolved spectrophotometric observations of DQ Her obtained in May 2002 with the $180 \mathrm{~cm}$ Rosino telescope. We analyze the evolution of the $\mathrm{H}_{\alpha}$ emission line profile throughout the eclipse of the WD and the accretion disk by the secondary. The observations are presented in Sect. 2. The results are discussed in Sects. 3 and 4.

\section{The observations and data reduction}

Time resolved spectroscopy of DQ Her was performed on May 19, 2002 with the $182 \mathrm{~cm}$ Rosino telescope at Mt. Ekar (Asiago, Italy). We used the AFOSC camera equipped with the Volume Phase Holographic grism VPH4. Eighteen spectra were obtained with exposure times of $360 \mathrm{~s}$. The covered spectral range is $6410-7018 \AA$ and the resolution is $0.7 \AA$. The journal of the observations is given in Table 1 where the orbital phases are given assuming the orbital period derived by Zhang et al. (1995) and a $T_{0}$ corresponding to the eclipse minimum of Fig. 2 (see next section).

Flat field images and photometric standard stars were also secured. Data reductions were performed using the standard IRAF software package. The eclipse and the out-of-eclipse spectra of the nova are shown in Fig. 1 where only the spectral range with the $\mathrm{H}_{\alpha}$ and the He I $\lambda 6678$ emissions is shown.

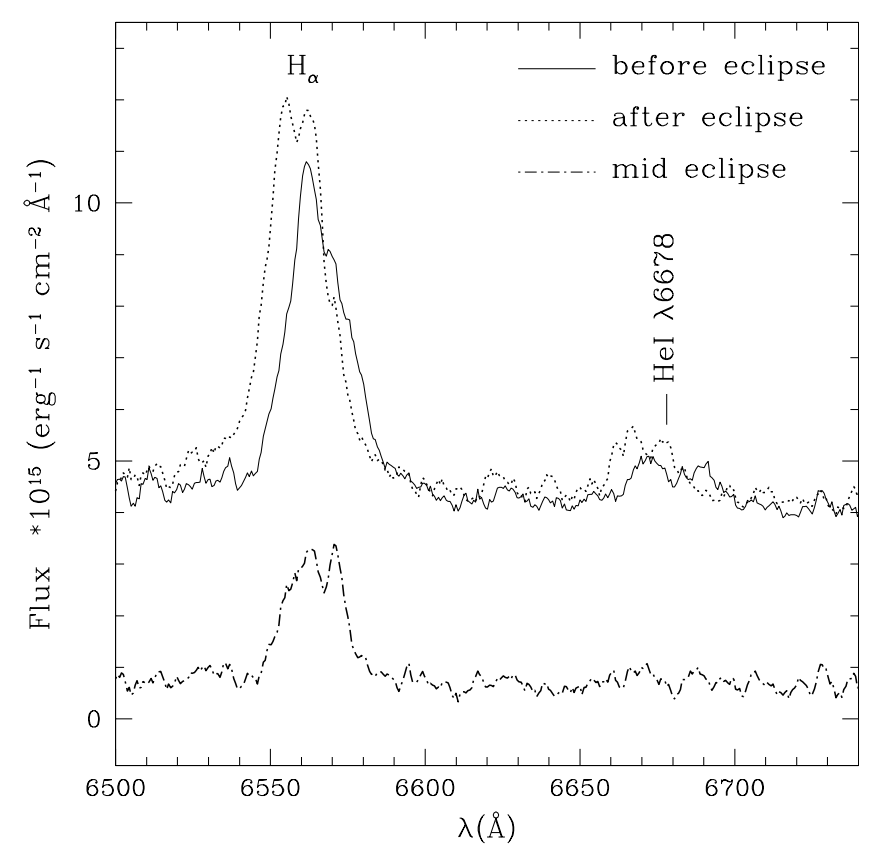

Fig. 1. The spectrum of DQ Her with the two main emission lines observed: $\mathrm{H}_{\alpha}$ and $\mathrm{HeI} \lambda 6678$. The dotted line is the mean of spectra s1 and s2 (Table 1) taken immediately before the eclipse ingress; the solid line is the the mean of post-eclipse spectra s14, s15, s16 and s17, taken between orbital phases $0.15 P$ and $0.24 P$; the dotted-dashed line is the mid-eclipse spectrum $\mathrm{s} 7$.

The light barycenter of the emission lines are clearly redshifted before the eclipse and blueshifted after the egress.

\section{The eclipse of the continuum}

\subsection{The $\mathrm{O}-\mathrm{C}$ diagram}

In Fig. 2 we show the eclipse light curve of the old nova in the flux of the continuum underlying the center of the $\mathrm{H}_{\alpha}$ emission. The flux and its error were estimated in each spectrum performing several linear fits of the continuum around the emission line. To determine the epoch of the minimum we fitted the data points with a spline function. Considering the errors associated to the fluxes of the continuum (vertical error bars in Fig. 2), we find that the eclipse minimum occurred at HJD $2452413.5482 \pm 0.0005$, the maximum error being eight times smaller than the integration time of individual spectra.

Using the eclipse linear ephemeris derived by Zhang et al. (1995)

$T_{\min }=$ HJD $2434954.94429( \pm 8)+0.1936208964( \pm 12) E$

the mid-eclipse time of Fig. 2 corresponds to an $\mathrm{O}-\mathrm{C}=+0.0013 \pm 0.0006 \mathrm{~d}$, that is a $1.9 \pm 0.9 \mathrm{~m}$ positive time delay. The same result is obtained using the updated ephemeris given by Ogloza et al. (2000). This value is in agreement with the prediction that the orbital period of DQ Her is cyclically oscillating as a result of the presence of solar-type magnetic cycles in the secondary star (Applegate \& Patterson 1987; Warner 1988; Bianchini 1987, 1990). In fact, our results suggest that the $\mathrm{O}-\mathrm{C}$ diagram currently tends to a positive maximum. This should correspond to a minimum in the stellar magnetic activity and to a relative minimum in the 


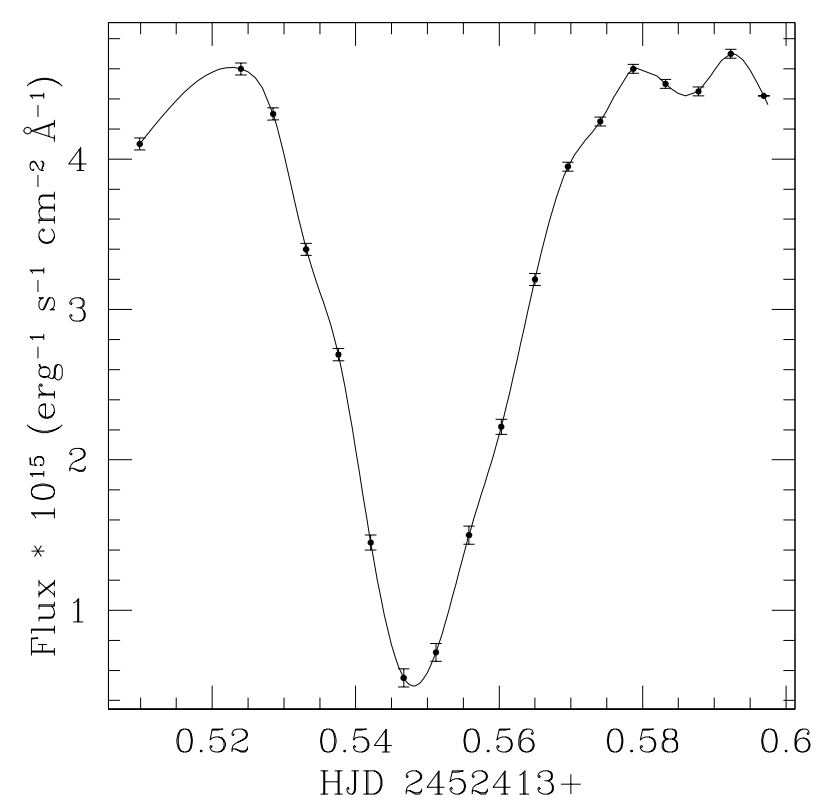

Fig. 2. The eclipse light curve of the nova derived from the continuum underlying $\mathrm{H} \alpha$ is represented by the spline function (solid line) fitting the data points. Vertical error bars are rather small. The profile is asymmetric as the ingress is $\sim 20 \%$ steeper than the egress. There seems to be no evidence of a luminosity bump prior to the eclipse ingress suggesting the presence of a hot spot. However, the poor phase coverage makes this conclusion rather uncertain.

mass transfer rate from the secondary. Since the previous O-C maximum occurred around JD 2444500 (see Zhang et al. 1995, their Fig. 4), the cycle we are presently observing might present a time scale slightly longer than $22 \mathrm{yr}$. However, the 14 yr period suggested by Warner (1988) cannot be excluded on the basis of fragmentary observations. In any case, the occurrence of a longer cycle might represent a secondary harmonic or stochastic behavior.

\subsection{The eclipse profile}

In the asymmetric profile shown in Fig. 2 there is no clear evidence of a luminosity bump preceding the eclipse ingress, but the absence of a classical hot spot remains uncertain due to the poor phase coverage. However, the eclipse ingress is steeper than the egress suggesting that the barycenter of the continuum emission is displaced towards the hot spot region. Actually, in several eclipse light curves of DQ Her reported in the literature (see, for example, Walker 1956, 1958), the hot spot seems to make only a small contribution to the luminosity of the old nova, which is optically dominated by the accretion disk. Alternatively, the hot spot might be centered right on eclipse. Walker (1956) also noticed that the light curve after the eclipse was often more luminous than before the eclipse ingress. Photo electric observations performed by Nather \& Warner (1969) in 1968, instead, show a bump before eclipse ingress. Multicolor observations by Zhang et al. (1995) also suggested a hot spot at orbital phases $\phi=0.8-0.9 P$ that becomes less visible at increasing wavelengths. We notice that Zhang et al.'s observations, and probably also those of Nather \& Warner (1969), were performed in coincidence with minima in the O-C vs. JD diagram of Zhang et al. (1995). Minima should then correspond to maxima in the mass transfer rate as discussed in previous section. The eclipse profile of Fig. 2 shows no bump preceding the eclipse ingress. Thus, it is possible that during our observation the hot spot was faint. This might be consistent with our large positive $\mathrm{O}-\mathrm{C}$ value suggesting a relatively low mass transfer rate.

The eclipse depth shown in Fig. 2 is $2.40 \pm 0.05$ mag. This is $\sim 0.75$ mag larger than the $\sim 1.65$ mag found by Zhang et al. (1995, their Fig. 1) in the $R$ band for a 1991 observation. These authors noticed that the eclipse depth in $B$ had considerably increased with time from $\sim 1.0 \mathrm{mag}$ in 1954 to $\sim 2.7 \mathrm{mag}$ in 1992 . This was interpreted as due to the decreasing background luminosity of the expanding nova ejecta. For the same reason, we cannot directly compare the eclipse depth of Fig. 2 with that observed by Zhang et al. (1995) in the $R$ band. Monochromatic multi-wavelength eclipse depths corrected for the background nebula emission were given by Schneider \& Greenstein (1979). The depth at the $\mathrm{H}_{\alpha}$ wavelength is $2.69 \mathrm{mag}$, that is slightly larger than what we observed. We notice that Schneider \& Greenstein's observations were taken in 1978 when the eclipse $\mathrm{O}-\mathrm{C}$ values were fluctuating around zero, possibly during a relatively higher mass transfer phase of the binary system. In all cases, Schneider \& Greenstein (1979) demonstrated that the spectrum and the apparent monochromatic magnitude of the nova at mid-eclipse are consistent with an M 3.5 star seen at a distance modulus of +7.4 . The conclusion is that the continuum from the disk is totally eclipsed.

The duration in phase of the eclipse of Fig. 2 is from $0.88 P$ to $0.15 P$ that basically confirms the mean $\Delta \phi \sim 0.25$ width estimated from the literature. This width suggests a disk which extends up to $90 \%$ of the WD Roche lobe (Harrop-Allin \& Warner 1996). We recall the fact that the presence of a strongly elliptical disk, like the one obtained by the numerical simulations performed by Wood et al. (2000), might eventually produce variable eclipse widths.

\section{The emission lines}

\subsection{The fluxes and the equivalent widths}

Figure 3 shows the variations of the fluxes of $\mathrm{H}_{\alpha}$ and $\mathrm{HeI} \lambda 6678$ emission lines. The line emission regions are only partially obscured by the secondary. The eclipse depths correspond to a decrease of about $68 \%$ and $74 \%$ of the out-of-eclipse line intensity for $\mathrm{H}_{\alpha}$ and HeI, respectively. Martell et al. (1995) found that the eclipse depths of $\mathrm{H}_{\beta}, \mathrm{H}_{\gamma}, \mathrm{H}_{\delta}$ and $\mathrm{H}_{\epsilon}$ corresponded to a decrease of their intensities of about $74 \%, 83 \%, 84 \%$ and $87 \%$, respectively. The smaller depth observed in the lower Balmer lines and, in particular, in $\mathrm{H}_{\alpha}$ is explained by the presence of a consistent contribution from the background nebula and by a Balmer decrement that is steeper in the nebula than in the accretion disk. Young \& Schneider (1980) found that, at eclipse minimum, the nebular contribution to $\mathrm{H}_{\beta}$ was $\sim 50 \%$. Thus the nebular contribution in $\mathrm{H}_{\alpha}$ cannot be smaller than that.

The eclipse profiles of the two emission lines appear symmetrical between phases 0.88 and 1.08 , with a minimum at 


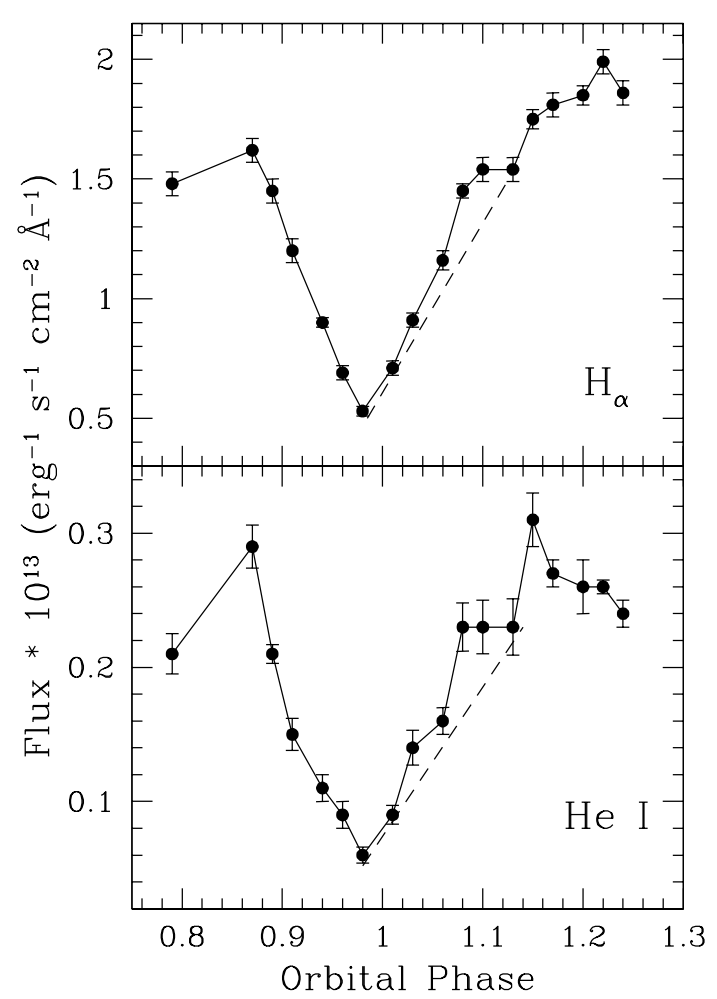

Fig. 3. The variations of the fluxes of $\mathrm{H}_{\alpha}$ and HeI 66678 . Phases are given with respect to the minimum observed in the continuum of Fig. 2. The minimum in the intensity of both the emission lines occurs at a phase $0.02 P$ before that of the continuum. In both cases, we notice the presence of a bump around phase $0.1 P$. The dashed lines put in more evidence the bumps showing that they might be interpreted as the result of a sudden steeper increase of the emission line intensities between phases $0.98 P$ and $0.1 P$.

phase 0.98. The eclipse seems to start at the same phase of the continuum but to end slightly before, so their minima precede that of the continuum by $0.02 P$. Petterson (1980) found that the center of the $71 \mathrm{~s}$ phase shift curve also falls at photometric phase $-0.02 P$. Hutchings, Cowley \& Crampton (1979), by measuring radial velocities outside eclipse, found that the stellar conjunction occurred at photometric phase $-0.04 P$. The latter result, although contradicted by spectroscopic observations performed by Young \& Schneider (1980), might suggests some occasionally more pronounced axial asymmetry of the disk causing a shift in the barycenter of the velocity field with respect to those of the continuum and of the emission lines. During the egress from the eclipse, the fluxes of the emission lines show a plateau between phases $0.08 P$ and $0.12 P$ followed by a sudden increase. This phenomenon was also observed by Martell et al. (1995). If we considered the plateau as part of the eclipse profile, the total eclipse duration would reach that of the continuum. Thus, as shown in Fig. 3, the plateau can be interpreted as a bump in the line intensity centered at $\phi \sim 0.1$. This might be caused by the sudden appearance of some localized optically thin region within the binary system possibly connected with the appearance of the blueshifted component of the emission profile as discussed in the next section.

The behavior of the equivalent widths of $\mathrm{H}_{\alpha}$ and HeIr6678 emission lines shown in Fig. 4 are due to the

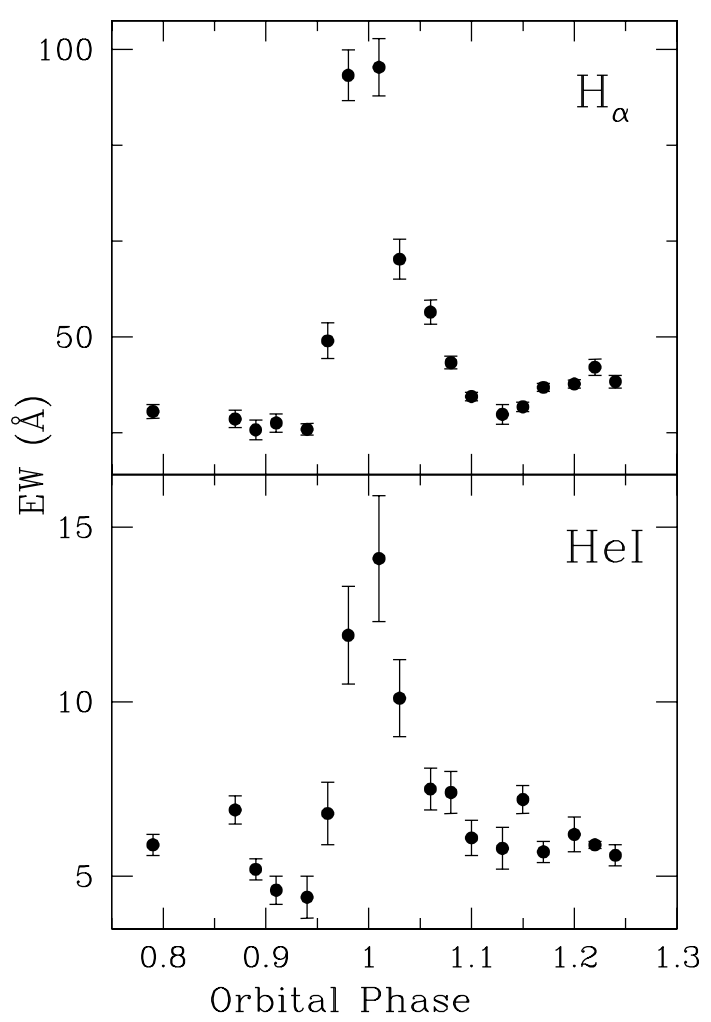

Fig. 4. The variations of the EW of $\mathrm{H}_{\alpha}$ and HeI throughout the eclipse. Phases are as in Fig. 3.

combined effect of the variations of the continuum and the emission lines. The equivalent widths show a peak at $\phi \sim 0.0$ because the eclipse occurs more in the continuum than in the emission lines. The asymmetry observed in Fig. 4 is due to the fact that, after mid eclipse, the emission lines become brighter faster than the continuum.

\subsection{The triple-peaked profile of the emission lines}

Figure 5 shows the evolution of the $\mathrm{H}_{\alpha}$ profile throughout the eclipse. The observed behavior is typical of an eclipsing accretion disk in that the blue wing disappears and reappears before the red one. The profiles present three main peaks labelled a, $\mathrm{b}$ and $\mathrm{c}$, centered at $\lambda \lambda 6555.5,6562$ and 6570.5 , respectively. The wings of the line profiles extend up to $\pm 1200 \mathrm{~km} \mathrm{~s}^{-1}$ suggesting the presence of a "broad component". The radial velocities of the three peaks remain almost unaltered. A proof is that the sum of all the spectra still shows the triple peaked profile as if these components were not affected by the orbital motion. As we will show, radial velocity variations are detected only in the broad component of the emission lines.

It is worthwhile to recall that Martell et al. (1995) suggested that some of the line doubling at all phases is likely due to the background nebula and that an important contribution to the emission lines must also come from the inward-facing hemisphere of the secondary star.

The central component " $\mathrm{b}$ " of the $\mathrm{H}_{\alpha}$ profile is close to the rest wavelength of the line and is eclipsed by about $62 \%$ of its maximum intensity. This emission component might originate near the $L_{1}$ Lagrangian point and/or the surface of the 


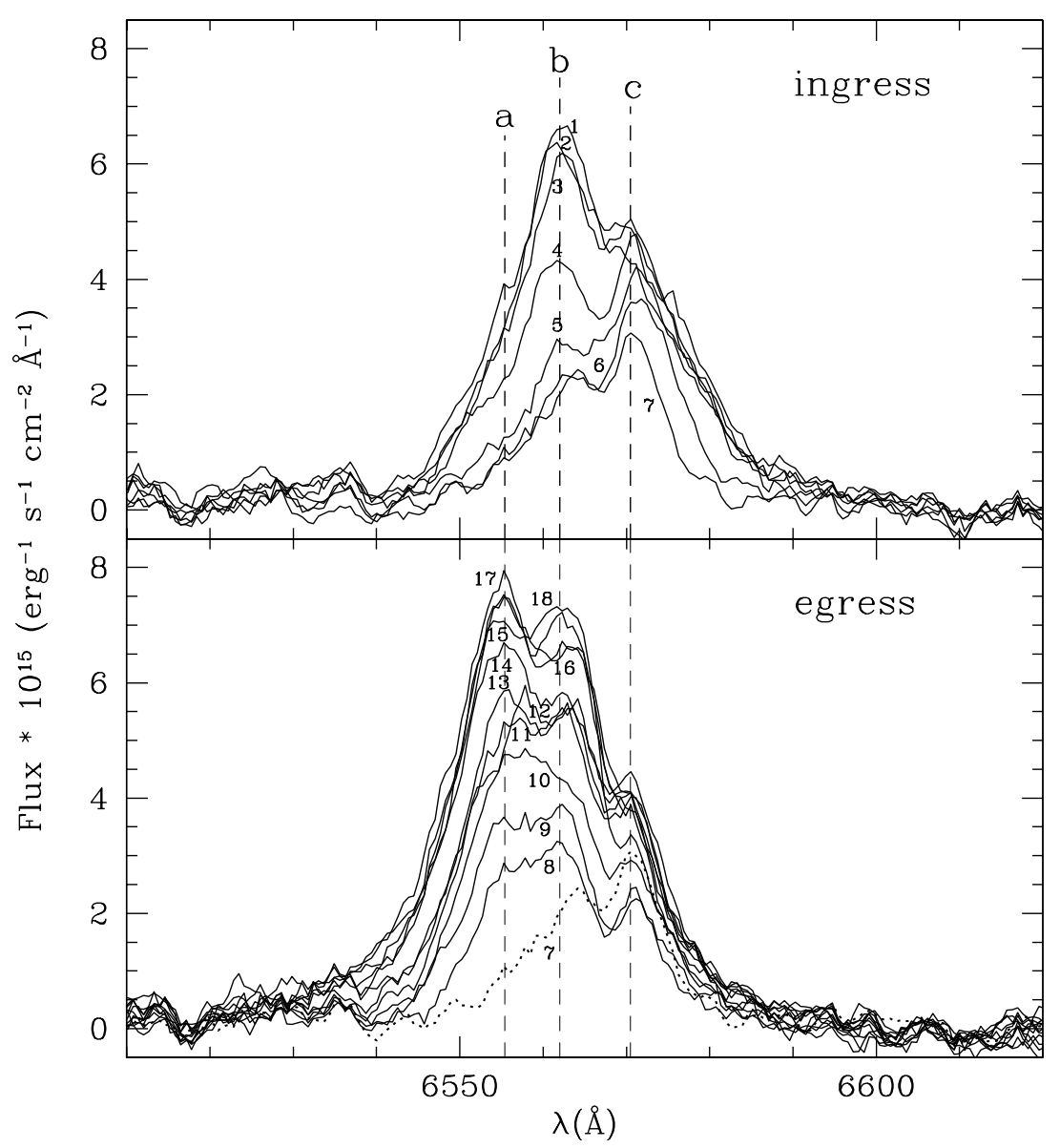

Fig. 5. The sequence of the $\mathrm{H} \alpha$ profiles entering and exiting the eclipse. The spectra are normalized to the continuum. The temporal sequence of the profiles is indicated by progressive numbers as in Table 1 . Components $\mathrm{a}, \mathrm{b}$ (which corresponds to the rest wavelength) and $\mathrm{c}$ are marked by vertical dashed lines. No radial velocity variations are shown by the three main peaks, but only drastic changes in their relative luminosities. Actually, only the wings of the lines seem to be affected by the orbital motion.

secondary facing the WD. However, since it is not totally eclipsed, at least some of the emission might come from an extended corona, a small circumstellar shell and/or a wind from the accretion disk. The latter hypothesis reminds the case of the dwarf nova IP Peg that also showed emission line profiles with a stationary zero-velocity component ascribed to an outflowing wind (Piché \& Szkody 1989). Marsh \& Horne (1990) specified that the low-velocity emission component seen in IP Peg should be produced by a spherical low-velocity wind close to the WD in order to comply with the constraints from the eclipse.

The blueshifted component "a" indicates a radial velocity of $-297 \mathrm{~km} \mathrm{~s}^{-1}$ and is the only emission peak to undergo an almost total eclipse. It appears weak and poorly resolved before the eclipse but it becomes quite prominent after the egress, contributing to the observed post-eclipse bump of the emission line intensity. A tentative explanation might be as follows. When part of the accretion stream, after the impact with the disk outer rim, flies above the disk surface, it follows a ballistic trajectory towards the hot magnetic WD. Since for $i \sim 86.5^{\circ}$ we can see only the region of the disk opposite to the WD, the accretion flow downstream the hot spot would become visible only around orbital phase $0.15 P$, just while it is moving towards us.
The redshifted component "c" corresponds to a radial velocity of $+388 \mathrm{~km} \mathrm{~s}^{-1}$ and is more marginally affected by the eclipse $(\sim 50 \%)$. The presence of this stationary redshifted emission explains why the red wings of the line profile (i.e. the broad component) show smaller wavelength changes than the blue ones. Such an emission might be produced by a receding gaseous knot of the nova shell. However, inspection of our CCD frames shows that the emission feature does not extend beyond the PSF profile of the star. An alternative explanation might be represented by the presence of a gaseous jet from the system, although such an hypothesis would deserve some physical justification.

The sequence of the HeI $\lambda 6678$ line profiles entering and exiting the eclipse is shown in Fig. 6. Due to the low signal to noise ratio of individual profiles, we plotted averages of two or three successive spectra of Table 1 . We still notice the presence of three main peaks again labelled $a, b$ and $c$. The structure of the HeI emission profile is however complicated by the fact that the trough that separates components $b$ and $c$ tends to dip below the continuum during the eclipse almost suppressing the central component $b$. This tells us that an absorption feature, perhaps due to the secondary, is present. Peak $b$ at $\lambda 6677$ could represent, like in $\mathrm{H}_{\alpha}$, the zero velocity component of the 


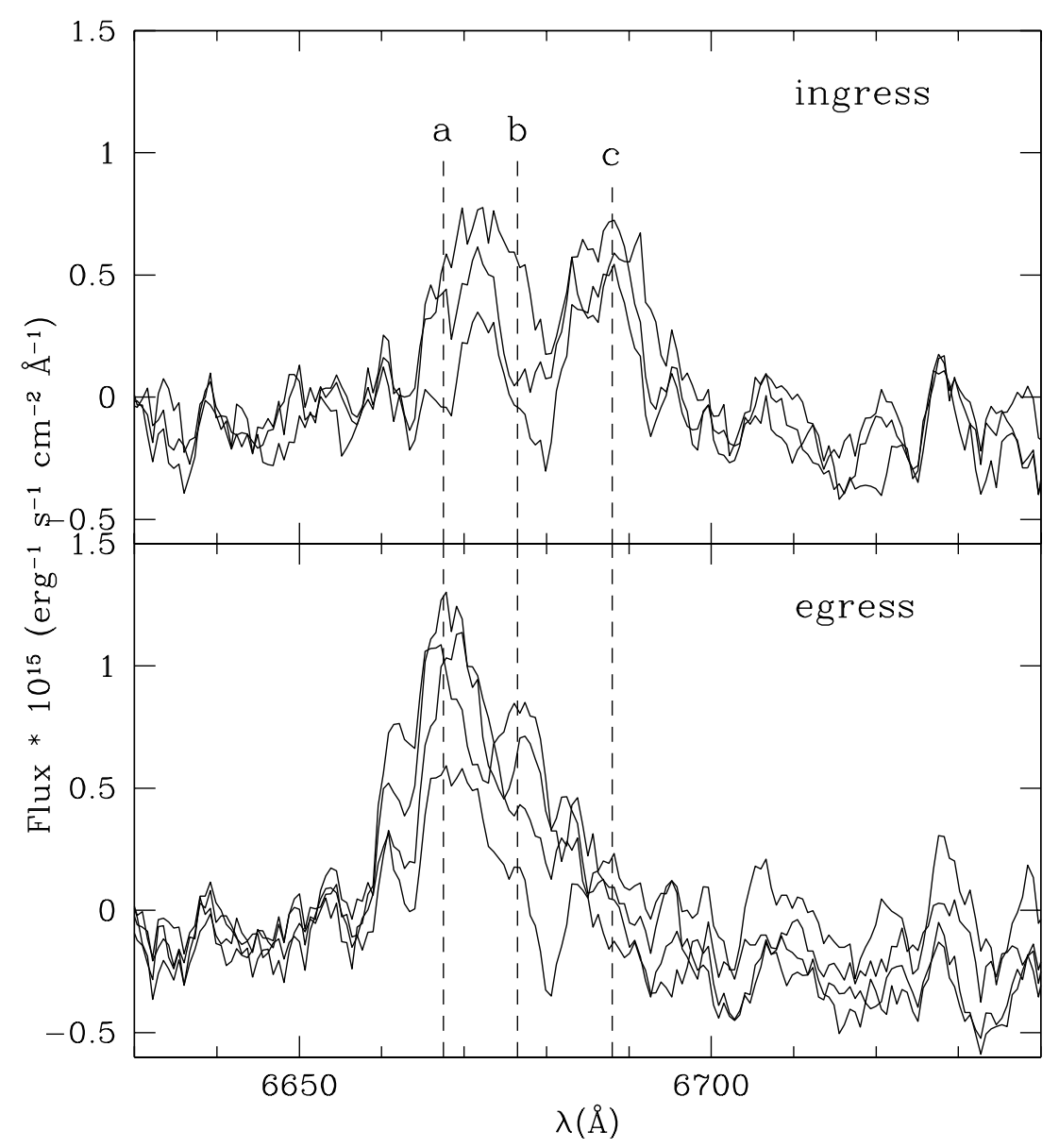

Fig. 6. The sequence of HeI 26678 profiles entering and exiting the eclipse. Due to the low signal to noise ratio of individual spectra, the temporal sequences of the line profiles are now represented by averages of two or three successive spectra of Table 1 . Component $a$, component $b$ and component $\mathrm{c}$ are marked by vertical dashed lines.

emission. The blueshifted component a of the HeI emission, like that of $\mathrm{H}_{\alpha}$, becomes prominent only after the eclipse and indicates a negative radial velocity of about $-472 \mathrm{~km} \mathrm{~s}^{-1}$. The redshifted component $\mathrm{c}$, at $\lambda 6688$, indicates a positive radial velocity of about $+494 \mathrm{~km} \mathrm{~s}^{-1}$ that is practically symmetrical with respect to the blueshifted component. Figure 3 shows that the eclipse depth of the HeI emission is larger than that of $\mathrm{H}_{\alpha}$ suggesting a smaller contribution from the nebula. We also notice that the radial velocities indicated by the HeI line profile are quite larger than those of $\mathrm{H}_{\alpha}$ and are more consistent with the velocity field of the material in the accretion disk.

\subsection{Radial velocity curves}

The radial velocities of main peaks a, b and c of Fig. 5 do not show significant variations through the eclipse. Their mean velocities plotted in Fig. 7 confirm the absence of orbital motion effects. Figure 7 shows that orbital motion is however detected from the broad component of the $\mathrm{H}_{\alpha}$ emission by taking the means of the wavelengths of the wings at an intensity level above the continuum corresponding to $1 / 5$ line maximum. The radial velocity curve of the broad component shows the rotational disturbance and suggests $K_{1} \sim 140 \mathrm{~km} \mathrm{~s}^{-1}$, consistently with the more precise determination obtained by
Horne et al. (1993). Hutchings et al. (1979) found a barycentric velocity of $-60 \mathrm{~km} \mathrm{~s}^{-1}$ while Fig. 7 indicates a systemic velocity around $+50 \mathrm{~km} \mathrm{~s}^{-1}$. This is however explainable as the stationary redshifted component $\mathrm{c}$ covers the red wing of the broad component of the emission line any time it shifts towards shorter wavelengths.

Due to the poor signal to noise ratio, we didn't try to obtain a radial velocity curve from the broad component of the HeI emission.

\section{Conclusions}

\subsection{Cyclical variations of the mass transfer rate}

The $\mathrm{O}-\mathrm{C}=0.0013 \mathrm{~d}$ derived from the eclipse of Fig. 2 follows and confirms the cyclical behavior of the $\mathrm{O}-\mathrm{C}$ vs. JD diagram shown by Zhang et al. (1995) suggesting a main secular periodicity somewhat larger than 22 yr. According to Applegate \& Patterson (1987) and Warner (1988), the orbital period variations should be anti-correlated with the variations of the mass transfer rate produced by solar-type magnetic cycles of the secondary. Thus, the epoch of our observation should be near a relative minimum of the mass transfer rate in DQ Her. This hypothesis is perhaps supported by the lack of a well pronounced hot spot preceding the eclipse of the continuum and 


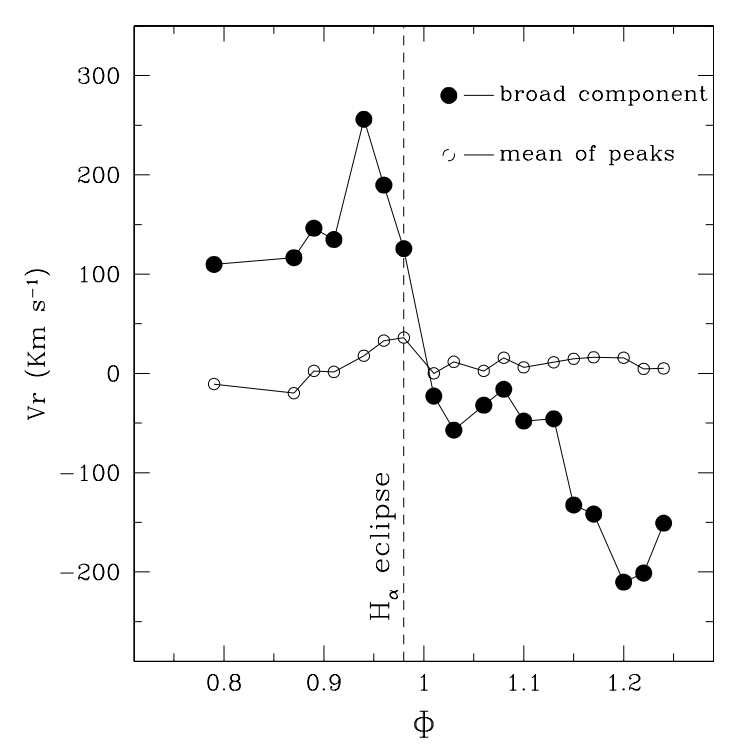

Fig. 7. The mean radial velocities of components $\mathrm{a}, \mathrm{b}$ and $\mathrm{c}$ of the $\mathrm{H}_{\alpha}$ emission are represented by filled circles. No significant radial velocity variations are seen. The orbital motion is reflected only by the broad component of the line, by taking the position of the wings at $20 \%$ height maximum. The effects due to the rotational disturbance are clearly seen.

by the $\mathrm{H}_{\alpha}$ eclipse depth being $\sim 0.29$ mag smaller than reported by Schneider \& Greenstein (1979).

\subsection{The line emitting regions in $D Q$ Her}

The evolution of the $\mathrm{H}_{\alpha}$ and the HeI $\lambda 6678$ emission profiles is reminiscent of an eclipsing accretion disk. However, most of the profiles are characterized by the presence of three main peaks, labelled $\mathrm{a}, \mathrm{b}$ and $\mathrm{c}$ that show little or no radial velocity variations but only changes in their relative intensities. The central component $b$ corresponds to the zero-velocity wavelength of the lines, while components a and c are roughly symmetrical with respect to it. Such a triple-peaked profile is more evident in $\mathrm{H}_{\alpha}$ because the HeI emission is weak and also complicated by the presence of a strong absorption component. The radial velocities of the side components of the HeI emission are rather symmetrical and might be consistent with the Keplerian velocities in the accretion disk. The suspicion that the bulk of the $\mathrm{HeI}$ emission is produced in the accretion disk is also supported by the fact that its eclipse depth is larger than that of $\mathrm{H}_{\alpha}$, the latter being more significantly contaminated by the background expanding nebula.

The $\mathrm{H}_{\alpha}$ emission profile is better resolved and its evolution throughout the eclipse better defined. Its side peaks a and $\mathrm{c}$ show lower radial velocities than $\mathrm{HeI},-300 \mathrm{~km} \mathrm{~s}^{-1}$ and $+390 \mathrm{~km} \mathrm{~s}^{-1}$, respectively. The extended wings of the line profile suggest the presence of a broad component that might correspond to the actual emission line from the accretion disk. We have suggested that the presence of the zero-velocity component $b$ might have different explanations: i) an emitting region near the $\mathrm{L}_{1}$ Lagrangian point; ii) an emission from the surface of the secondary facing the WD; iii) a wind perpendicular to the surface of the accretion disk. he presence of stationary emission components is not new amongst CVs. In particular, we might envisage some similarities with the dwarf nova IP Peg that, during its optical outbursts, also showed a central feature in the emission line profiles attributed to an outgoing wind emanating from the central regions of the accretion disk (Piché \& Szkody 1989; Marsh \& Keith 1990).

The blueshifted component a is the only one that undergoes an almost total eclipse. We suggest that it can be produced by the ballistic accretion flow downstream the hot spot that can be seen only past the eclipse, when it is moving towards us above the region of the concave disk opposite to the WD.

The redshifted component $\mathrm{c}$ is more marginally affected by the eclipse and the peak is well pronounced also at minimum intensity. The emission seems to be confined within the PSF of the star. The peak might then be produced by a small receding gaseous knot of the nova shell or by a receding jet of material from the nova, the latter hypothesis being purely speculative.

Our spectra do not cover an interval of orbital phases larger than $0.24 P$. We conclude that further good quality timeresolved spectroscopy performed throughout full orbital cycles is necessary to cast some more light about the nature of the emission lines structures of DQ Her. We also suggest that the analysis of high resolution $\mathrm{H}_{\alpha}$ frames of the old nova taken with HST might give information about the possible presence of a circumstellar nebula that would support the wind interpretation of some of the observed spectral features.

\subsection{The orbital motion}

Radial velocity changes produced by the orbital motion of the WD are detected only in the broad component of the emission line, that is from the blue and red wings of the line profiles. They show the rotational disturbance and confirm the $K_{1}=140 \mathrm{~km} \mathrm{~s}^{-1}$ measurement given by Horne et al. (1993). The constant presence of the redshifted nebular component $\mathrm{c}$ prevents the red wing of the broad component from being seen at its most blueshifted position. This explains the positive barycentric velocity shown in Fig. 7.

Acknowledgements. We are very indebted to an anonymous referee for the precious comments and suggestions that quite improved this paper. A.B. wishes to thank the people of the Department of Physics and Astronomy of the University of Wyoming for their friendship and support. This work has been supported by NSF REU Grant AST 9732039, and by the Italian MURST.

\section{References}

Africano, J. L., \& Olson, E. C. 1981, PASP, 93, 130

Applegate, J. H., \& Patterson, J. 1987, ApJ, 322, L99

Bianchini, A. 1987, Mem. SAI, 58, 245

Bianchini, A. 1990, AJ, 99, 1941

Chanan, G. A., Nelson, J. E., \& Margon, B. 1978, ApJ, 226, 963

Duerbeck, H. W. 1987, Space Sci. Rev., 45, 1

Duerbeck, H. W. 1999, IBVS, 4731

Harrop-Allin, M. K., \& Warner, B. 1996, MNRAS, 279, 219

Horne, K., Welsh, W. F., \& Wade, R. A. 1993, ApJ, 410, 357

Hutchings, J. B., Cowley, A. P., \& Crampton, D. 1979, ApJ, 232, 500

Livio, M. 1992, ApJ, 393, 516

Marsh, T. R., \& Horne, K. 1990, ApJ, 349, 593 
Martell, P. J., Horne, K., Price, C. M., \& Gomer, R. H. 1995, ApJ, 448, 380

Mukai, K., Still, M., \& Ringwald, F. A. 2003, ApJ, 594, 428

Nather, R. E., \& Warner, B. 1969, MNRAS, 143, 165

Nelson, M. R., \& Olson, E. C. 1976, ApJ, 207, 195

Ogloza, W., Dròzdz, M., \& Zola, S. 2000, IBVS, 4877

Orio, M., Covington, J., \& Ögelman, H. 2001, A\&A, 373, 542

Patterson, J. 1994, PASP, 106, 209

Petterson, J. A. 1980, ApJ, 241, 247

Piché, F., \& Szkody, P. 1989, AJ, 98, 2225

Robinson, E. L. 1976, ApJ, 203, 485

Schneider, D. P., \& Greenstein, J. L. 1979, ApJ, 233, 935

Silber, A. D., Anderson, S. F., Margon, B., \& Downes, R. A. 1996a, ApJ, 462, 428
Silber, A. D., Anderson, S. F., Margon, B., \& Downes, R. A. 1996b, AJ, 112, 1174

Smak, J. 1980, Acta Astron., 30, 267

Starrfield, S. 1989, in Physics of Classical Novae, ed. A. Cassatella, \&

R. Viotti (New York: Springer Verlag), Proc. IAU Coll., 122, 127

Walker, M. 1954, PASP, 66, 230

Walker, M. 1956, ApJ, 123, 68

Walker, M. 1958, ApJ, 127, 319

Warner, B. 1988, Nature, 336, 129

Wood, M. A., Simpson, J. C., Kawaler, S. D., et al. 2000, Baltic Astronomy, 9, 211

Young, P. J., \& Schneider, D. P. 1980, ApJ, 238, 955

Zhang, E., Robinson, E. L., Stiening, R. F., \& Horne, K. 1995, ApJ, 454,447 